\title{
E1 USALI y la historia de los sistemas uniformes de coste: ¿Un reto hispano?
}

Francisco Martin Peña ${ }^{1}$, Ramon M. Soldevila de Monteys $^{2}$ (D), Vanesa Berlanga Silvente ${ }^{3}$ ii

${ }^{1}$ Universitat Oberta de Catalunya (Spain)

${ }^{2}$ Universitat Abat Oliba CEU (Spain)

${ }^{3}$ Universitat de Barcelona (Spain)

fmartin@uoc.edu,rsoldevilad@uao.es, berlanga.silvente@ub.edu

Received October, 2016

Accepted November, 2016

\section{Resumen}

Objeto: Este trabajo tiene por objeto efectuar una indagación sobre la evolución histórica del movimiento uniformizador en contabilidad de costes y su posición actual en el sector del alojamiento. El objeto primordial es 'aprender del pasado', al tiempo que se plantea una cuestión de futuro: ¿hacia dónde deben apuntar las actuales propuestas de gestión de costes en la industria hotelera? El objetivo concreto de este trabajo, se fija, sobre la base de una necesaria mirada retrospectiva, en perfilar y analizar cuáles son en la actualidad las necesidades informativo - contables de los actuales complejos hoteleros hispanos, a la luz de los dos grandes objetivos perseguidos por el pensamiento contable a lo largo de su (ancha) historia en busca de la uniformidad. Una uniformidad que desde 1926 han venido persiguiendo las sucesivas versiones del USAH o el acrónimo actualmente convertido en USALI, Uniform System of Accounts for Lodging Industries.

Diseño/metodología/enfoque: Este trabajo se compone de dos partes diferenciadas y claramente interconectadas: 
- En una primera investigación se examina el movimiento 'uniform system’ en su época más dinámica, como fueron los años de entre guerras (1920 - 1940), sus objetivos, aportaciones más destacadas, entorno económico y político, influencias.

- En una segunda indagación este estudio se sitúa en la época actual y concretamente en la industria hotelera española, donde, mediante el método de la encuesta, se pretende evaluar e interpretar el grado de necesidad percibida por los agentes directivos del sector de un movimiento contable uniformista a la española.

Aportaciones y resultados: La indagación ofrece dos tipos de aportaciones y resultados: por un lado, una reconfiguración del papel desempeñado por el movimiento uniformador en la historia de la contabilidad de gestión. En segundo lugar, se evidencian mediante un estudio de campo cuáles son las principales necesidades informativas del sector hotelero en España, más allá de una mera adaptación sectorial del plan general de contabilidad vigente.

Originalidad / Valor añadido: Los autores han basado su trabajo en las grandes líneas evolutivas que han fructificado en la diferentes versiones del USALI, considerando su utilidad como punto de partida para la elaboración de un sistema de gestión contable ajustado a las necesidades e idiosincrasia del sector hispano. Entre las conclusiones que pueden extraerse, tanto de la revisión del pasado como del análisis de las necesidades del presente, está la evidencia que la industria hotelera europea se enfrenta a una asignatura pendiente que la tradición norteamericana ha sabido encauzar de una forma comparativamente más avanzada: disponer de un instrumento completo y uniforme de información contable.

Palabras clave: Empresas hoteleras, Plan contable sectorial, Sistemas uniformes de costes, Indicadores de gestión hotelera

Códigos JEL: M10, M13, M15

Title: American USALI and the historical evolution of the uniform cost systems: A Spanish challenge?

\section{Abstract}

Purpose: This study presents an inquiry on the historical evolution of the uniforming movement in cost accounting and its current position in the lodging sector. Its paramount objective is 'to learn from the past' and, at the same time, to pose a question of future: where 
are aiming the current techniques of cost management in the lodging industry to? A specific purpose of this inquiry was fixed, on the base of a necessary retrospective look, in outlining and analyzing the actual accounting informative needs of today's hotel complexes, considering the two main directions that hotel accounting has taken throughout its (long) history in search of uniformity. A uniform purpose that, from 1926 on, the successive versions of the USAH Uniform System of Accounts for Hotels has being pursuing (an acronym modified in 1996 as USALI - Uniform System of Accounts for Lodging Industries).

Design/methodology: This work composes of two parts differentiated and clearly interconnected:

- In a first study it examines the movement uniform system in its most dynamic period, as they were the years between the two WW (1920 - 1940), its aims and most outstanding contributions, its economic and politician circumstances, social influences, etc.

- In a second study the interest is focused in the current period and specifically in the Spanish lodging industry, where, by means of the method of the survey and personal interviewing, it aims to evaluate and interpret the degree of need perceived by the managerial agents of the sector about an accounting uniform movement in Spain.

Findings: The inquiry offers two types of contributions and results: on the one hand, a reconfiguration of the role exerted by the 'uniforming' movement in the history of management accounting; on the other hand, it makes evident, by means of a field inquiry, which are the main informative needs of the lodging sector in Spain, beyond a mere sectorial adaptation of the current general plan of accounting.

Originality/value: The authors have focused their inquiry on the big evolutionary lines that have given fruit in the different versions of the USALI, considering its utility as the starting point for a hypothetical system of accounting management adjusted to the needs and idiosyncrasy of the Hispanic sector. One of the main conclusions that can be extracted is that, either by looking at the review of the past or by summarizing the analysis of the present needs, the evidence shows that the European hotel industry faces a pending task that the North American tradition has been successful to channel in a way comparatively more advanced: the fact of having a complete and uniform instrument of accounting information.

Keywords: Hotel costs, Chart of accounts, Uniform systems of costing, Hotel management metrics 
Jel Codes: M10, M13, M15

\section{Introducción}

La primera parte del artículo pretende únicamente ofrecer un estudio no exhaustivo de lo que fue en los años 1920 y 1930 todo un movimiento innovador en el pensamiento y las prácticas contables. Pretende subrayar no sólo la intensidad sino también las líneas, unas acertadas y otras no tanto, por las que según los autores de la época debía discurrir la 'nueva' contabilidad. En esta línea se hace hincapié en que tanto el pensamiento contable francés como el norteamericano buscan soluciones contables de tipo interno, antes de nada. Sea la polémica gala en torno al 'prix de revient', sean los 'uniform systems' anglosajones, cualquiera de las propuestas de solución que en esos años se conciben se centran en suministrar información de costes útil para el gobierno económico de las entidades que integran un sector. Se observa cómo dichas soluciones intentan cubrir dos necesidades informativas principales:

- Un sistema continuamente mejorado que proporcione información para la dirección de la entidad (objetivo interno);

- Un sistema uniforme que garantice la existencia de unas prácticas en el sector basada en la razonabilidad y la mejora continua (objetivo sectorial).

Esta primera parte del estudio concluye con una breve descripción de la evolución histórica del sistema uniforme mundialmente más destacado en hostelería: el sistema USALI y sus once versiones (de 1926 a 2014). En este punto no sólo interesa resaltar los contenidos que se han venido agregando o modificando al sistema sino también, y considerando el objeto de la segunda parte de este estudio, las circunstancias en que dentro del sector han tenido lugar (asociaciones responsables, progresiva aceptación mundial, comisiones de seguimiento, conexiones con los organismos de contabilidad y auditoría externas, etc.).

La segunda parte, describe los resultados del estudio empírico (encuesta), con el fin de señalar cuáles son las necesidades informativas que el directivo hotelero español considera prioritarias, no tanto para generar datos contables de destino público como para disponer de información que contribuya a la mejora del establecimiento (objetivo interno) y la comparabilidad de dichos datos (objetivo sectorial). Las conclusiones de esta limitada pero estricta indagación empírica conducen de forma natural al 
planteamiento de la cuestión que es el objeto fundamental de esta aportación: ¿qué reacción merece el movimiento 'uniform system' al sector hotelero español? ¿Conviene adherirse a la filosofía subyacente del modelo USALI? Y en caso afirmativo, ¿qué actitud adoptar, la de aceptar lo que este modelo ya ofrece en su última edición, o adoptar su metodología a la hora de crear un sistema propio español?

\section{E1 movimiento Uniform System en las dos décadas de entreguerras}

En los dos subapartados que siguen se realiza una incursión en la historia del pensamiento contable de dos décadas al inicio del siglo $\mathrm{XX}$, al tiempo que se destacan algunos hechos suficientemente significativos para la comprensión de la tendencia. Para obtener un retrato de la época más ceñido, el estudio examina las corrientes 'uniform' en dos zonas geográficas igualmente destacadas para la historia de la contabilidad en aquella época: el movimiento uniforme en Francia, el movimiento uniforme en EE.UU y donde a estos dos campos de investigación se añade una breve alusión a la evolución histórica del USALI a lo largo de sus once versiones sucesivas, comenzando por la primera en el año 1926.

\section{1. “Prix de revient” y sistemas uniformes de costes en Francia}

En la Francia de entreguerras, a semejanza de lo que sucedió especialmente en EE.UU., se dieron cita una serie de hombres de empresa y del pensamiento administrativo destinados a dejar una huella notable en la historia de la gestión empresarial europea. En cuanto a los comienzos de esta primavera del pensamiento empresarial galo quizás pueda señalarse un pistoletazo de salida en 1889 año de la exposición universal y de la construcción de la emblemática Torre Eiffel en Paris.

El año de la muerte de Henry Fayol (1925) estaban vigentes y brillaban con luz propia nombres como el de A. Detoeuf fundador y presidente de Alsthom, el coronel E. Rimailho, ex-militar e incansable profesor, o el ingeniero industrial A. Citroën. Tres nombres suficientes para evidenciar el espíritu de una época que ellos mismos calificaron de crucial:

Auguste Detoeuf (1983-1947) es también autor de una obra de aforismos y pensamientos de gestión, Propos d'O.L. Barenton, confiseur (1947), que todavía se reedita en Francia. Rechazó los planteamientos liberales de Ludwig von Mises y fue cofundador de la revista Nouveaux Cabiers que propugnaba, bajo premisas sociales cristianas, la aproximación entre trabajadores y patronal. 
Emile Rimailho (1864-1954) ingeniero militar, se especializó en la fabricación y mejora de piezas de artillería. Se le atribuye la introducción en Francia de las piezas estándar de recambio, a propósito de la fabricación del fusil Lebel. En 1913 se licencia del ejército para dedicarse a la dirección empresarial y a la docencia, siendo nombrado administrador de la CGCEM - Compagnie Générale de construction et d'entretien du matériel de chemin de fer.

André Citroën (1878-1935) es celebrado tanto por su espíritu innovador en la industria automovilística (viajes a Detroit) como por sus originales acciones de marketing (entre ellas destaca, por su grandiosidad la propaganda que en 1925 la torre Eiffel hizo de su apellido leído en vertical a base del empleo de varios centenares de miles de bombillas).

En 1937 A. Detœuf, aprovechó la ocasión que le brindaba la solicitud de prologar el Rapport sobre las prácticas y cálculo uniforme del coste de producción completo (“prix de revient”) en Francia. Es en este informe preconizado por el citado ex-lugarteniente coronel Rimahillo, entonces presidente del organismo patronal CEGOS, donde Detœuf propone un afortunado 'apólogo' que conviene recordar aquí con sus palabras:

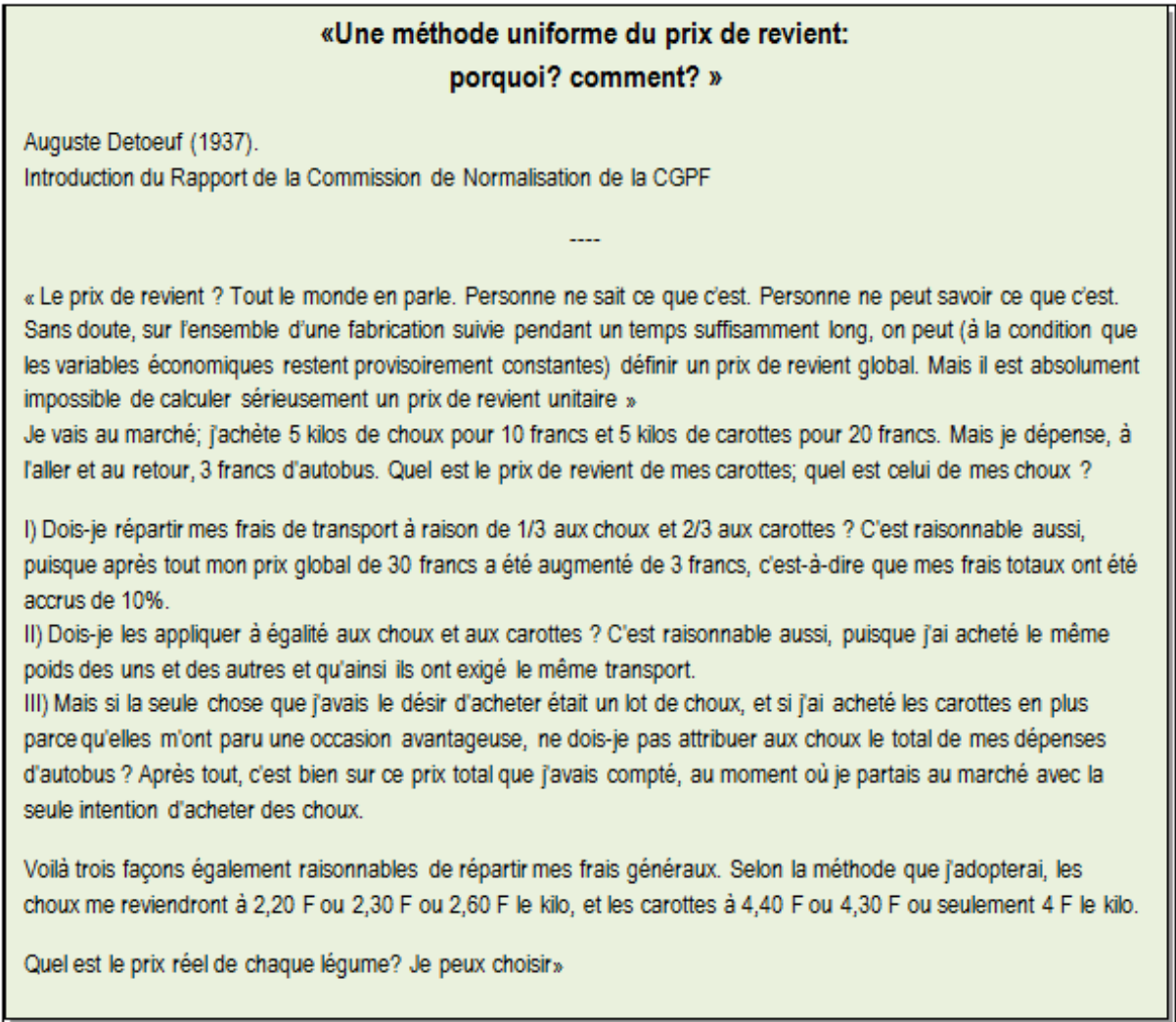

Figura 1. Apólogo Detoeuf (1937) de los costes indirectos 
Con una admirable economía narrativa y de datos, Detoeuf presenta la figura de un vendedor que va al mercado a comprar $5 \mathrm{~kg}$ de coles por 10 francos y acabó comprando también $5 \mathrm{~kg}$ de zanahorias por 20 francos. El problema contable lo plantean los costes 'indirectos' representados en esos 3 francos que le costó el desplazamiento. ¿Cómo asignar dichos costes al coste final de las coles y/o de las zanahorias? Un problema clásico con varias soluciones.

El apólogo, sin embargo, no concluye aquí. Una vez visualizada la dificultad de concretar el verdadero coste completo (prix de revient) de un producto, el autor plantea la cuestión principal: ¿cómo afecta la ambigüedad del cálculo del coste al mercado del sector? Para esta segunda parte de su breve 'cuento', el directivo francés se vale de un segundo agente:

"Maintenant, nous sommes deux, Pierre et moi à avoir fait les mêmes opérations en vue de revendre nos choux et nos carottes, avec un bénéfice de 10\%. Mais nous avons choisi chacun une méthode différente pour calculer nos couts de revient. Quels prix de vente allons-nous proposer? Que vont faire nos clients qui veulent acheter $10 \mathrm{~kg}$ de choux et $10 \mathrm{~kg}$ de carottes? Quelles seront les conséquences sur nos bénéfices? Et si Pierre et moi avions adopté la même méthode de calcul des couts de revient?"...

El cliente -concluye Detoeuf- comprará todas las coles que el autor puso en venta, puesto que éste eligió el primer método y sobre la base de ese cálculo puede ofrecerlas al precio más bajo. Similarmente el cliente comprará las zanahorias de Pierre, por haber seleccionado el tercer método y ofrecer las zanahorias más baratas del mercado:

Ingresos Autor+Pierre $=34.10$ f, si Autor adopta método I y Pierre método III .

Ingresos Autor+Pierre $=36.30 \mathrm{f}$, si ambos hubieran adoptado el método I.

El mensaje normalizador es inequívoco: en lugar de discutir sobre la mayor o menor razonabilidad de determinadas asignaciones de costes, los directivos harán bien en cuidar que en su sector todos basen sus cálculos sobre métodos uniformes. Estamos ante una palpable muestra de lo que pretendía la corriente francesa a favor de los sistemas uniformes de costes. En realidad, el movimiento uniformista en Francia data de bastantes años atrás de 1937. De hecho A. Detoeuf escribe el prólogo sobre la versión definitiva de un Rapport que la CGPF - Confédération Génerale de la Production Française, fundada en 1926, había encargado a una comisión CGOST - Commission Générale d'Organisation Scientifique du Travail, recién creada en el seno de esa confederación y hoy bien conocida por su nombre más popular de Cégos. 
En realidad el Rapport auspiciado por Rimailho se publicó por primera vez en julio de 1928 y en él ya se abogaba por el empleo uniforme (y universal) de un método de asignación de costes indirectos basado en las denominadas 'secciones homogéneas'. Ya en un Informe previo de 1923 se hacía alusión al método de las secciones homogéneas aunque con una denominación que no hizo fortuna (sections d'ateliers). Marc Nikitin (1992) fija la década de los años 1820 como la época en que este concepto comenzó a debatirse en Francia, y considera la obra de Guibault, Traité de comptabilité et d'administration industrielles (1865), clave para su difusión en distintos sectores.

En línea con lo apuntado, puede afirmarse, con Henri Bouquin que a lo largo del primer tercio del siglo XX, se generó en Francia un potente movimiento normalizador, en el que uno de los puntos estrella se centró en el concepto de 'prix de revient' y el tratamiento de los costes indirectos mediante un método de segmentación por talleres. En su opúsculo La comptabilité de gestion (Col: «Quesais-je? » n 3175. PUFPresses Universitaires de France) afirmaba que:

"La méthode divise les ateliers et les services de l'entreprise en "sections homogènes" selon un principe sans doute bérité des travaux allemands et peut-être néerlandais, practiqué par Ramailho à la Compagnie génerale de construction et d'entretien du matériel, CGCEM, qui assurait l'entretien et le maintenance des matériels ferroviaires de la COmpagnie Paris-Orleans et de la compagnie PLM" (pp. 26) (Bouquin, 2003).

Como puede intuirse en el apólogo de Detoeuf una de las metas prioritarias era que el sector generara información comparable, teniendo como medida de base el concepto de 'prix de revient' o coste final de un producto o servicio ya en manos del cliente. Ese coste final debía ser calculado según un método uniforme denominado de las 'secciones homogéneas' conforme indica Palewski (1944), L'organisations cientifique du travail, Col: "Quesais-je? » $n^{\circ}$ 31, (pp. 45). Pero a la hora de establecer casos ejemplares el historiador se encuentra con dos interesantes extremos: el del mismo Taylor recomendando a la Betlehem Steel que siguiera el ejemplo de las compañías ferroviarias; y el que iba implícito en la crítica acerba que Joseph Proudhon (1855) dirige contra los cálculos de estas mismas compañías (ver Bouquin, 2003)

Por otra parte, atendiendo a Nikitin (1992), La naissance de la comptabilité industrielle en France, Tesis doctoral, Université Paris-Dauphine, los debates sobre la conveniencia de adoptar un principio generalizado en la asignación de los costes indirectos aparecen en Francia por los años 1830 y pueden ejemplificarse en los casos de dos empresas emblemáticas: Saint-Gobain y DecazevilleSait-Gobain fue creada en 1665 (Luis XIV) como fábrica de vidrios (actualmente de materiales para estructuras de alto rendimiento). Su sistema contable de costos ha sido recientemente analizado a causa de las novedades 
que tan tempranamente adoptó, incluido un original sistema retributivo. Por su parte, Decazeville fue fundada a mediados del siglo XIX, por el Duque Decazes, productora de minerales y metales. Su sistema contable ha sido parangonado con el papel análogo jugado por Springfield Armory en los Estados Unidos.

Un estudio de Dragos (2009), (Les multiples enjeux d'une technique de gestion :pratiques et discoursdans la répartition des fraisgénéraux, Université d'Orléans: tesis doctoral, pp. 79), destaca el hecho de que a partir de 1860 los intercambios comerciales entre Francia e Inglaterra se liberalizan de tal modo que el reto competencial implica un interés creciente por los métodos y prácticas de la contabilidad industrial y el tratamiento de los costes indirectos.

Como se deduce del mismo apólogo de Detoeuf, a dicho tratamiento se confía durante el periodo de entreguerras dos objetivos principales:

- La correcta gestión de la empresa basada en datos de coste comparables que permita el diseño de estrategias de precios y de control de costes.

- La garantía de un sector libre de empresarios 'proletarios' que, ignorando las leyes del mercado, lo destruyen a la postre sin saberlo.

En los años de la Ocupación estos objetivos se mezclaron con el intento por parte del Estado de controlar la producción sector por sector, siguiendo las líneas sugeridas en un discurso del mariscal Pétain. El movimiento de los sistemas uniformes de cuentas no contemplaba sin embargo esta 'perversión' del concepto en el que los autores más destacados siguieron creyendo en el papel clave que un coste calculado de manera uniforme juega en el progreso comercial de un país.

Una fe en el coste uniforme que llegó a inspirar afirmaciones tan sorprendentes como ésta:

"La connaissance exacte du prix de revient et l'étude des facteurs qui déterminent celui-ci forment la base sur laquelle reposent tous les principes directs ou indirects de l'organisation du travail." (Trossen, 1934).

Un primer dogma (el de la mejora de la organización del trabajo) al que se añadía un segundo 'artículo' de esa misma fe (Zimnovitch, 1997) resumido así por Calmès (1922):

Il est inutile d'insister ici sur la grande importance du prix de revient pour l'industrie. Obtenir des prix de revient aussi détaillés et aussi exacts que possible est non seulement une question vitale pour chaque fabrique en particulier, mais aussi une question d'intérêt général qui entraîne des conséquences économiques et sociales de la 
plus haute importance (avilissement des prix, concurrence déloyale et faillites par suite de prix de revient incomplets et inexacts; salaire à la tâche, participation aux bénéfices, échelle mobile des salaires et autres moyens d'entente entre le capital et le travail sont basés sur les prix de revient).

El mismo Zimnovitch (1997) observa cómo, poco a poco y de manera indirecta, la exaltación de la centralidad del coste de producción en la economía, eleva la consideración del papel social que juegan los contables y la contabilidad. Según el autor Edouard Julhiet (1922):

"La connaissance du prix de revient est indispensable au chef d'entreprise; l'établissement du prix de revient est un des principaux services que rend la comptabilité."

No es de extrañar que el paso siguiente a los intentos de uniformizar el concepto fuera la de reglamentar las prácticas de cálculo y empleo. Acabada la II Guerra Mundial la aventura de un sistema uniforme de contabilidad de costes desemboca en Francia en la aprobación del Plan de Contabilidad de 1947, donde aparece, aunque opcional, el método de las secciones homogéneas.

Este método de las secciones homogéneas se mantiene en el Plan de 1957 y en el de 1984, aunque con una denominación significativamente distinta: centres d'analyse en lugar de sections homogènes. El actual Plan Contable francés no incluye metodología alguna, como la que el coronel Rimailho y el mismo Detoeuf consideraban un típico fruto de la inventiva francesa y Zemnovitch, por el contrario, califica de método "bien banal".

Por último se debe observar que esta nota histórica quedaría incompleta si no se hiciera alusión a los influjos del exterior en estos años en el pensamiento francés. Y más en concreto, de los EE.UU.

La fascinación que los métodos utilizados por determinados sectores estadounidenses (automovilismo, ferrocarril, fundición) inspiran a los directivos franceses puede deducirse del modo cómo se elevó a categoría la simple anécdota de un fundidor de Pittsburgh. Este empresario anglosajón señalaba a sus colegas galos el modo cómo él solía tratar a la competencia (Zimnovitch, 1997). Y concluía así:

J'ai offert à tous mes concurrents d'installer chez eux des systèmes de prix de revient, car à essayer de concurrencer actuellement leur devis imprécis, basés sur des prix de revient faux, je perds plus d'argent que je n'en dépenserais pour leur payer des experts de prix de revient. 


\section{Origen y evolución de los 'sistemas uniformes de costes' en los EEUU}

Según Gerald Berk (1997), fue en las dos primeras décadas del siglo XX (años 1910 y 1920) cuando se produjeron los progresos más destacados en contabilidad de gestión en EE.UU. La clave estuvo en que las numerosas asociaciones empresariales de la época (funderías, ferrovías, caucho....) aceptaron también ellos, al igual que los colegas franceses, el 'artículo de fe' según el cual las prácticas contables uniformes eran garantía de progreso en los dos frentes ya señalados: el buen control de gestión interna de una firma y la ausencia de empresarios ignorantes. Fueron las asociaciones de principios de siglo las que potenciaron las prácticas contables de costes de sus antecesoras decimonónicas de la manera más sorprendente. Los dos grandes males contra los que se luchaba de forma asociada eran la conducta ignorante y el intrusismo. Para este autor, gracias al movimiento uniformista

"Associationalists made more progress than commonly thought in confronting the two obstacles said to undermine their project: malfeasance and illegitimacy. Moreover, under the tutelage of twentieth-century associafionalists, nineteenth-century managerial accounting endured and progressed in surprising ways."

El mismo Berk (1997) subraya el papel desempeñado por dos grandes asociaciones de la época: la entonces llamada Asociación Nacional de Contables de Costes, fundada el año 1919 en Buffalo (NY) (NACA-National Association of Cost Accountants; hoy, IMA-Institute of Management Accounting), cuya misión principal consistió en hacer progresar la teoría y práctica contables útiles para la gestión, y la fijación de un "Standard Cost-FindingSystem" a la manera como estaban perfilando las asociaciones de impresores. En esta época de entreguerras se suscita en EE.UU. un debate que, examinado desde la perspectiva histórica, puede considerarse providencial para el progreso de los sistemas uniformes en general. Es la disputa en torno a los costes por intereses. El cruce de argumentos sobre este punto resultó de extraordinaria utilidad puesto que a la hora de tomar conciencia de la propia posición se perfilaba con mayor nitidez el importante papel que un sistema uniforme de costes puede llegar a desempeñar en una economía como la norteamericana.

A caballo entre los dos siglos tuvo lugar en los EE.UU. una extensa ola de fusiones empresariales. El fenómeno agudizó los desacuerdos entre los partidarios de una posición y los de la contraria. Por ambos extremos emergieron con inusitada pasión y tomaron partido sean los defensores de la solución técnica o de los denominados ingenieros, sean los que se inclinaron por la solución económica o de los auditores contables. Incluir o no incluir como coste del producto los gastos por intereses, esa era la cuestión. 
Johnson y Kaplan (1987) señalan cómo entre los auditores contables prevaleció la opinión de que dichos costes por intereses debían excluirse del cálculo del coste del producto (p. 130 s.). Esta disputa se desarrolló en el seno del AIA - American Institute of Accountants y podía seguirse en la revista de la asociación Journal of Accountancy. Esta asociación creó una comisión a fin de emitir una opinión autorizada sobre la cuestión. En 1918 ya estaba pronto el informe para su comunicación en el congreso de ese año. En él, tras señalar lo espinoso del problema se consideraba finalmente que los intereses no son "gastos de producción" sino "beneficios anticipados" y como tal no "tienen cabida lógica en el cómputo del coste de producción”, lo que significa que su inclusión llevaría a engaño las cifras del balance y de los resultados (Berk, 1997).

El informe abordaba también la posibilidad de crear una cuenta de reserva mientras no se perfeccionara la venta del producto y por tanto el resultado. La opinión final sobre esta solución fue que resultaba innecesariamente compleja y redundante. El rechazo a la opinión de los técnicos inspira estas líneas finales del informe:

"In conclusion, it is the opinion of your committee that the inclusion in production cost of interest on investment is unsound in theory and wrong, not to say absurd, in practice." (AIA-American Institute of Accountants, 1918, pp. 110-112)

Berk sigue narrando los principales acontecimientos de esta disputa añadiendo que al poco tiempo de la emisión de este informe, la Asociación de Instructores Universitarios de Contabilidad promovió un debate entre dos de los expertos más significados: Scovell, favorable a la inclusión y Haney, contrario a la misma. Interesa especialmente la valoración de este último. Para Haney, la razón más profunda para estar en contra de la inclusión de los intereses es el amor a la verdad, y el rechazo del engaño. Haney no se queda aquí y su reflexión siguiente merece la cita textual. En primer lugar, conviene no descartar motivos menos inocentes:

Other motives exist, however, which maybe more sinister. During the last few years, there has been a general tendency in the business world to eliminate competition in price. We have an organized propaganda for the maintenance of resale prices on manufactured articles. We find many accountants standing for the value of investment, instead of the cost of investment as the basis for price fixing...

En segundo lugar, determinados fenómenos pueden dar que pensar sobre los verdaderos objetivos perseguidos en esta cuestión: 
Also the portentous growth of association in all the industries is a wellknown phenomenon; and these associations generally center in the idea of what is called "uniform systems of costaccounting," a phrase which may mean systems of uniform costs. Now comes an effort to put fixed return on investment into cost. Where will it all end? Are we to reach some advanced economic stage in which the "industrial engineer's" art will enable the tired business man to sell "at cost" and still receive a return on investment? (American Association of University Instructors, 1919, pp. 38-39).

La fundación de la NACA en 1919 pretendía encontrar un camino de en medio entre los defensores de la contabilidad de costes basada en la verdad y las opiniones más pragmáticas de los que preocupaba sobre todo la difusión de soluciones contables con un lenguaje común. Así en 1921 esta asociación se aliaba con la ASME-American Association of Mechanical Engineers para elaborar un "Lexicon of Common Terms". Por otra parte se creó un boletín oficial de carácter mensual y un departamento de investigación. En 1922 el entonces director de dicho departamento, J.P. Jordan, ya afirmaba que entre el conjunto de materiales recogidos hasta el momento los más valisosos son "the uniform cost systems devised by trade associations" (Berk, 1997). El Departamento había recogido ya más de un centenar de sistemas de costes de las asociaciones. En 1924, siendo ya presidente de la asociación, afirmaba en un congreso organizado por la Cámara de Comercio, y ante un nutrido grupo de fabricantes: "cooperation is necessary: manufacturers must get together with one another and with accountants".

Esta cooperación entre los fabricantes y los contables, entre el mundo profesional y el universitario, se prefijó un objetivo básico de partida: ya era un buen progreso encontrar un idioma único de los negocios. Como señala Berk, el copresidente del Cracker Cost Club, de la Asociación de Galletas y Biscotes (Cracker and Biscuit) Harry Green fue quien mejor resumió esta necesidad en el encuentro organizado por la NACA en 1921:

"The point, which impressed upon me more vividly than ever before, is the great need for all of us to speak the same language. I believe that most misunderstandings in our work come about because we do not all speak the same language... If we were all to get down to using a common language and to calling the same things by the same names, I believe about seventy-five per cent of our troubles would be eliminated" (NACA Yearbook, 1921, pp. 163-64)

Por su parte, Nielsen analizó en 1966 el contenido de un documento de 1919 que ya formulaba una queja en torno a la falta de un lenguaje común en los sectores. El texto, formulado por la asociación de pasteleros del Medio Oeste era suficientemente expresivo. En él podía leerse la siguiente apreciación: 
«No quiere decir prácticamente nada el que un fabricante diga a otro que su margen bruto es, o debería ser, un determinado porcentaje de sus ventas, puesto que ese otro fabricante ignora lo que eso significa, salvo que sepa cómo el primer fabricante ba calculado su coste y margen bruto. Por consiguiente, los fabricantes podrían estar hablando idiomas distintos cuando emplean términos como beneficio bruto y coste, a menos que se aseguren de que todos ellos entienden los componentes del coste de una forma similan»

El otro punto de escándalo era la dispersión de los precios. La Cámara de Comercio estadounidense ofrecía datos punzantes. Según las estimaciones en 66 ciudades las diferencias en el precio de un encargo tipográfico era un $72 \%$ de media y en fundición maleable podía llegar a ser hasta del 122\%, en caucho del 100\% y en seda, del 125\% (Chamber of Commerce, 1924).

Se esperaba del cálculo y de la contabilidad uniforme del coste la posibilidad de frenar la tentación de bajar los precios conociendo bien los costes. Por otra parte podría servir para justificar los precios delante de los clientes a la hora de negociar con ellos.

Además de fomentar la fiabilidad de las comparaciones con datos de costes de un mismo sector y los datos temporales de una misma empresa, Jordan señalaba un beneficio interno especial y extremadamente beneficioso: las rivalidades se desplazarán del mercado a la fábrica, y en concreto a la calidad del producto y al beneficio obtenido con la reducción de costes así analizados.

"Uniform cost accounting was, in a (non-pejorative) sense, a panacea - a program intended to improve the capacity of business firms to apprehend theirperformance, and to communicate internally and with one another." (Berk, 1997, pp. 240).

Ya adelantamos que entre las asociaciones que se significaron por estos años a la búsqueda de un sistema uniforme de costes había que destacar a la Asociación de Impresores. Su interés por el sistema uniforme les llevó a construir ya en 1909 una comisión la APCC - American Pinters' Cost Commission, más tarde recreada como UTA - United Typothetae of America. En 1916 anunciaba un plan trianual para difundir el que denominaron su UTA Standard Cost-Finding System, que fue periódicamente revisado todavía en las décadas posteriores.

De este sistema uniforme para impresores interesa resaltar aquí el uso estricto de la denominada "departamentalization". La apuesta se cifraba en identificar los departamentos que se consideren necesarios en un negocio de impresión. Por lo general se distinguían tres divisiones departamentales: composición, impresión y encuadernación, a los que podían agregarse otros como el de corrección de pruebas, gestión de materiales o comercialización(UTA Standard Cost Finding System, 1927). Dentro de 
estos grandes grupos departamentales cabía la posibilidad de desglosar los mismos según el grado de detalle y complejidad que se deseara dar al sistema en función del tratamiento que se le pretendía dar al factor humano y a la recogida de datos.

Puede verse cómo el efecto positivo que se espera en las personas y en el grado de precisión de los cálculos del coste es prácticamente el mismo en los 'departamentos' norteamericanos que en las 'secciones homogéneas' francesas. El otro principio, de uniformidad, también se comparte en ambos mundos: se busca un "Uniform Cost-Finding System", que haga comparables las cifras del sector y de un año respecto al siguiente. Y resulten creíbles los cálculos y la información de los denominados "costes medios departamentales" con vistas a su mejora gradual.

\section{Origen y evolución del USALI}

La última versión publicada del USALI es la número 11 y fue publicada en mayo de 2014 para su entrada en vigor a principios de 2015 (Schmidgall, 2014). Con esta ocasión el EI-Educational Institute (Instituto de Educación) publicó en su página web una entrevista con el vicepresidente de la Comisión de Gestión Financiera del AH\&LA - American Hotel and Lodging Association. En ella se podía leer:

The primary purpose of the Uniform System of Accounts for the Lodging Industry is to provide operating statements that are formatted to provide hotel owners, managers, and other interested parties with information and data that is pertinent to the unique operating environment of the lodging industry.

Como puede verse la versión más reciente también se adhiere al primero de los dos grandes objetivos tradicionales del movimiento uniforme: obtener y proporcionar información útil para el gobierno del hotel teniendo en cuenta el singular entorno que hoy día impregna la industria del alojamiento.

The Eleventh Revised Edition of the Uniform System of Accounts for the Lodging Industry was revised to reflect changes in industry practice and to address contemporary industry practices, including but not limited to: 1 Technology updates; 2 Sustainability; 3. Globalization; 4. New Terminology; 5. Enhanced Ratio Analysis.

Entre los contenidos que la nueva versión USALI ha renovado y enriquecido ostensiblemente está el uso de las métricas o indicadores de gestión. Así, ya en la quinta edición (1952) se aconsejaba, desde luego, el uso de dichas métricas, es decir, la elaboración de: 
"Sufficient statistical information to aid the management in arriving at sound conclusions as to the efficiency of the operation of the various departments".

Pero la propuesta se solventaba en unos cuantos párrafos de texto y formulario. Sin embargo, en la última versión se dedican hasta 42 páginas. En conjunto, mientras la $8^{a}$ edición se editaba como un sobrio volumen de 139 páginas, la siguiente alcanzaba las 238, mientras que la $10^{\text {a }}$ edición contenía 275 y la $11^{\text {a }}, 353$ páginas. He aquí el año de aparición de cada una de las 11 ediciones: $\left(1^{\mathrm{a}}, 1926\right)\left(2^{\mathrm{a}}, 1933\right)$ $\left(3^{\mathrm{a}}, 1939\right)\left(4^{\mathrm{a}}, 1946\right)\left(5^{\mathrm{a}}, 1952\right)\left(6^{\mathrm{a}}, 1961\right)\left(7^{\mathrm{a}}, 1977\right)\left(8^{\mathrm{a}}, 1986\right)\left(9^{\mathrm{a}}, 1996\right)\left(10^{\mathrm{a}}, 2006\right)\left(11^{\mathrm{a}}, 2014\right)$.

En los dos subapartados anteriores el análisis histórico estaba orientado a recrear los primeros años en que las economías modernas sintieron necesidad de uniformizar su información contable. En medio de este ambiente revisionista apareció la primera edición del Uniform System of Accounts, el año 1926. La época de su creación y las existencias de distintas corrientes uniformistas explican que ese año surgiera una amplia propuesta de uniformización contable: desde las propuestas de un léxico común hasta la propuesta de cálculos de costes. El USAH surgió por iniciativa de la Asociación Hotelera de la ciudad de Nueva York (The New York Hotel Association) y tras varias reuniones en Chicago entre expertos contables y profesionales del ramo. Ya se ha indicado que una primera necesidad compartida era la de disponer una terminología común. De aquí que ya la primera versión del USALI venía dotado de un cuadro de cuentas (si bien, no con las correspondientes 'definiciones y relaciones' de cada cuenta, a la manera europea).Por otra parte, la evolución del sistema ha desembocado, por su parte, en un auténtico modelo de contabilidad de costes (basado en el control económico de los Departamentos). El USALI es, pues, desde su primer lanzamiento algo más que un Plan Contable a la manera como cristalizó en Francia o Alemania. Otras de sus características de origen fue su vocación universalista. Algunos años más tarde de su creación era aceptado como propio por la American Hotel \& LodgingAssociation como un estándar nacional para Estados Unidos y Canadá, y en 1961 apareció una adaptación del sistema a pequeños hoteles y moteles titulado Uniform System of Accounts and Expense Dictionary for Small Hotels, Motels and Motor Hotels" para miembros de la American Hotel and Motel Association.

No puede olvidarse la influencia propagadora de los grandes grupos hoteleros con sede en Estados Unidos. Las grandes firmas hoteleras, del tipo Inter Continental, Hilton, Sheraton, HolidayInn, Ramada, se mostraron unos fervientes seguidores del USALI a través de los cuales el modelo se propagó por toda Europa. La tesis doctoral de la profesora de la Universidad de Algarve, Faria (2012) ofrecía un cuadro detallado sobre la difusión del USALI, del cual se ha extraído esta tabla: 


\begin{tabular}{|l|c|c|c|r|r|}
\hline \multicolumn{1}{|c|}{ Autores } & País & $\begin{array}{c}\text { Método de } \\
\text { investigación }\end{array}$ & Población & $\begin{array}{c}\text { Número de } \\
\text { respuestas } \\
\text { (y \%) }\end{array}$ & $\begin{array}{c}\text { Grado de } \\
\text { utilización del } \\
\text { USALI }\end{array}$ \\
\hline $\begin{array}{l}\text { Kwansa and Schmidgall } \\
(1999)\end{array}$ & EUA & Cuestionario & Responsables financieros & $112(22 \%)$ & $78 \%$ \\
\hline Campa Planas (2004) & España & Cuestionario & $\begin{array}{c}\text { Cadenas hoteleras y hoteles } \\
\text { individuales }\end{array}$ & $27(77.1 \%)$ & $63 \%$ \\
\hline Pavlatos e Paggios (2007) & Grecia & Cuestionario & Hoteles & $85(58 \%)$ & $12 \%$ \\
\hline Persic e Jankovic (2010) & Croacia & Cuestionario & N.d. & $51($ N.d. $)$ & $84 \%$ \\
\hline Faria (2012) & Portugal & Cuestionario & $\begin{array}{c}\text { Hoteles de 3 y 4 estrellas - } \\
\text { Algarve }\end{array}$ & $66(74.2 \%)$ & $50 \%$ \\
\hline
\end{tabular}

Tabla 1.Grado de utilización USALI. Fuente: Faria (2012)

Llegando a la conclusión de una tasa media de utilización del USALI del 50\% en Europa, frente a su masivo empleo en EE.UU. Estos datos contrastan con el estudio de 2004 realizado por Fernando Campa según el cual el 100\% de las cadenas internacionales manifestaban su utilización frente al 62.5\% de las grandes cadenas españolas y sólo un 37.5\% de las cadenas españolas medianas (Campa \& Rebull, 2007).

El conocimiento de los planteamientos históricos de la corriente "uniform" propone al directivo y hombre de empresa actual un reto cuya formulación podría ser aproximadamente la siguiente:

Nuestros directivos españoles ¿deben adherirse a esta corriente? Y, en el caso afirmativo, ¿deberían aceptar el camino ya andado por iniciativas tan inveteradas como el USALI o crear, en la más estricta tradición geográfica, unos contenidos propios para esta corriente en nuestro idioma y suelo? 


\section{Objetivos del estudio empírico}

La finalidad de la investigación empírica que sigue es la de describir el nivel de utilidad que tiene el sector hotelero sobre el Plan General de Contabilidad versus USALI así como detectar los indicadores más utilizados.

\subsection{Metodología}

\subsubsection{Procedimiento}

La investigación realizada se puede enmarcar dentro de un estudio más amplio ex post facto de carácter descriptivo-comparativo donde se han analizado los datos procedentes de la encuesta online (instrumento de análisis) que integra la información sobre la contabilidad en el sector hotelero en Cataluña.

El cuestionario abordaba preguntas relativas al perfil, factores de su gestión a corto y largo plazo, así como, las técnicas de gestión utilizadas. Se propusieron un total de 20 indicadores - Margen Bruto porcentual de explotación GOPMR (Gross operating profit margin ratio). Ingreso por habitación disponible RRevPAR (Rooms revenue per available room), Ingreso total por habitación disponible Total RevPar (Total revenue per available room), Beneficio de explotación EBITDA (earns before interest, taxes depreciation assets), Beneficio porcentual de explotación EBITDA (earns before interest, taxes depreciation assets), Ingreso por cubierto AFC Average foodcheck, \% coste de alimentos FCP (Food cost percentage), \% de coste de bebidas BCP (Beverage cost percentage), \% de coste de personal LCP (Labor cost percentage), \% Ocupación OP (occupancy percentage), \% Clientes por habitaciones ocupadas (number of ghests per occupied room), Duración de la estancia media (Average length of stay), Número de servicios de desayuno, Número de servicios de almuerzo, Número de servicios de cena, Ingresos por servicio, Audiovisuales, Salones, Menú banquetes, Coffee breaks - y otros aspectos que se recogen en la encuesta en http://www.survio.com/survey/d/D9I8R1S5I7V9O2X4N. Se pretenden conocer las características propias del sector, y cuáles son los sistemas contables de gestión desarrollados en las diferentes organizaciones.

Las preguntas se desarrollaron partiendo de cuestionarios ya utilizados en otras investigaciones y adaptadas al caso catalán (Campa \& Amat, 2005; Campa \& Rebull, 2007). El cuestionario fue presentado, en primer lugar por un grupo de expertos compuesto por profesores especialistas en la 
materia analizada, en el campo estadístico y entre los directivos de empresas pertenecientes a la población seleccionada. Resultado de esta revisión, se realizaron las modificaciones recomendadas.

\subsubsection{Población}

La población de estudio está constituida por los 460 hoteles ubicados en Cataluña y a la encuesta han respondido un total de 44 establecimientos hoteleros. Se han obtenido un total de 44 respuestas lo que supone una muestra representativa, para un coeficiente de fiabilidad del $95.5 \%$ y un margen de error del +/- 10\%, trabajando con una relación de p y q del $50 \%$, que representa la situación más desfavorable.

\subsubsection{Análisis de datos}

Para el tratamiento y análisis de los datos se ha utilizado el programa informático PASW Statistics, en su versión 20.0. Se han realizado los siguientes análisis: descriptivos univariados de todas las variables implicadas, descriptivos bivariados para explorar la relación entre las variables, pruebas de normalidad y pruebas de contraste no paramétricas para determinar la significatividad de las diferencias encontradas.

\section{Resultados}

Para situar nuestro análisis correctamente, parece adecuado definir primero algunas características del perfil de los 44 hoteles participantes del estudio. Los datos indican que la muestra se divide en dos grupos adecuadamente representativos, el 50\% manifiestan ser independientes frente al otro 50\% que pertenecen a un Grupo Hotelero. Respecto a la categoría del hotel, el 68.2\% son hoteles de 4 y 5 estrellas frente a un 31.8\% que tienen 3 estrellas o menos. Mayoritariamente, un $93.2 \%$ de la muestra participante elabora los informes contables mensualmente.

En nuestra investigación se halla que la mayoría de los hoteles (86.4\%) utiliza el Plan General de Contabilidad, frente a un 9.1\% que trabaja además con USALI. En este sentido, la evidencia empírica ha señalado que cuando se les ha preguntado sobre si sería bueno que el sector hotelero tuviera un plan de cuentas propio a semejanza de otros sectores, el 68.2\% han manifestado que sería bueno para poder gestionar mejor los ratios de los hoteles así como para un mayor análisis de los costes de los departamentos. 
A pesar de que el 81.8\% manifiesta utilizar sólo un PGC donde se registran los asientos contables para llevar el control de gestión del establecimiento hotelero, la evidencia empírica señala que un 77.3\% utiliza la cuenta de resultados por departamentos para su gestión incidiendo en los ingresos y los costes de habitaciones, de comida y bebida y de otros departamentos. Alrededor de un $75 \%$ utilizan un sistema de costes directos basados por departamentos analizando por separado aquellos centros generadores de ingresos (alojamiento, restaurante, etc.) de aquellos que solo generan gastos (administración, recursos humanos, etc.).

En referencia al análisis de los 20 indicadores más utilizados por el sector hotelero participante, la evidencia empírica señala un uso mayoritario de todos los indicadores preguntados (en una escala de 1 a 5). Señalando la "Duración de la estancia media Averagelength of stay" (3.63) e "Ingreso total por habitación disponible Total RevPar (Total revenue per available room)" (3.88) como los que han obtenido una puntuación más baja.

Se presentan diferencias significativas respecto a la utilización de algunos indicadores cuando se compara la tipología de ser un hotel independiente o pertenecer a un Grupo Hotelero.

Los indicadores que presentan diferencias significativas respecto a quien los utiliza son: "el cálculo de costes más frecuente en el hotel es el relativo a los diferentes departamentos" (sig. 0.021) y "la cuenta de resultados de su hotel que utiliza para su gestión, es por departamentos, incidiendo en los ingresos y los costes de Habitaciones, de Comida y Bebida y de otros departamentos" (sig. 0.04). El 43\% de los hoteles independientes son los que manifiestan que utilizan más "el cálculo de costes más frecuente en el hotel es el relativo a los diferentes departamentos". Pero respecto a "la cuenta de resultados de su hotel que utiliza para su gestión, es por departamentos, incidiendo en los ingresos y los costes de Habitaciones, de Comida y Bebida y de otros departamentos" el 41\% de hoteles que pertenecen a un Grupo Hotelero son los que hacen un mayor uso.

En este sentido, no se presentan diferencias significativas entre la necesidad o no de disponer de un Plan de Cuentas sectorial y la utilización de los indicadores de gestión. 


\begin{tabular}{|l|r|}
\multicolumn{1}{l}{$\begin{array}{l}\text { Utilizan sólo un Plan General de Contabilidad donde se registran los asientos contables para llevar el } \\
\text { control de gestión del establecimiento hotelero }\end{array}$} & .900 \\
\hline $\begin{array}{l}\text { Utilizan un sistema de control de costes independiente de la contabilidad utilizada para las Cuentas } \\
\text { Anuales }\end{array}$ & .353 \\
\hline Utilizan un sistema de costes por departamentos basado en costes directos & .082 \\
\hline $\begin{array}{l}\text { Se analizan por separado aquellos departamentos o centros generadores de ingresos (alojamiento, } \\
\text { restaurante...) de aquellos que solo generan gastos (administración, recursos humanos...) }\end{array}$ & .150 \\
\hline El cálculo de costes más frecuente en el hotel es el relativo a los diferentes departamentos & .021 \\
\hline $\begin{array}{l}\text { La cuenta de resultados de su hotel que utiliza para su gestión, es por departamentos, incidiendo en los } \\
\text { ingresos y los costes de Habitaciones, de Comida y Bebida y de otros departamentos }\end{array}$ & $\mathbf{. 0 0 4}$ \\
\hline
\end{tabular}

Tabla 2. Contraste Chi-Cuadrado de la utilización del PGC y control de gestión respecto a la variable dicotómica: Hotel independiente vs Grupo hotelero

La evidencia empírica señala que no se presentan diferencias significativas (Tabla 3) entre pertenecer a un Grupo Hotelero o ser Independiente y el uso de los indicadores analizados. Ambos grupos utilizan una serie de indicadores no contemplados en el PGC.

\begin{tabular}{|l|r|r|}
\cline { 2 - 3 } \multicolumn{1}{c|}{} & \multicolumn{1}{c|}{$\begin{array}{c}\text { Media } \\
\text { (Desviación típica) }\end{array}$} & $\begin{array}{c}\text { Significación } \\
\text { p-valor }\end{array}$ \\
\hline Margen Bruto porcentual de explotación GOPMR (Gross operating profitmargin ratio) & $3.97(1.33)$ & .006 \\
\hline Ingreso por habitación disponible RRevPAR (Rooms revenue per available room) & $4.02(1.25)$ & .054 \\
\hline Ingreso total por habitación disponible Total RevPar (Total revenue per available room) & $3.88(1.24)$ & .072 \\
\hline Beneficio de explotación EBITDA (earns before interest, taxes depreciation assets) & $4.10(1.36)$ & .224 \\
\hline $\begin{array}{l}\text { Beneficio porcentual de explotación EBITDA (earns before interest, taxes depreciation } \\
\text { assets) }\end{array}$ & $4.07(1.27)$ & .270 \\
\hline$\%$ de coste de personal LCP (Labor cost percentage) & $4.44(0.99)$ & .374 \\
\hline$\%$ Ocupación OP (occupancy percentage) & $4.44(1.22)$ & .370 \\
\hline Duración de la estancia media Average length of stay & $3.63(1.32)$ & .649 \\
\hline
\end{tabular}

Tabla 3. Contraste Chi-Cuadrado de indicadores respecto a la variable dicotómica: Hotel independiente vs Grupo hotelero

\section{Conclusiones}

El reto uniformizador en la industria hotelera española es un dilema ya que el mundo contable hispano lo que se plantea no es si debe existir un sistema uniforme en la industria hotelera, sino qué conviene más, si aceptar las propuestas 'profesionales' disponibles o inventar una propia. La tesis doctoral de la profesora Vacas (1999) mostraba una indisimulada inclinación por la 'solución' francesa:

Una característica de la planificación contable francesa, ha sido la adaptación del Plan Contable General a los distintos sectores de actividad económica. Las adaptaciones sectoriales se autorizaron por una ley de 28 de 
diciembre de 1959 y se bicieron operativas a partir de un decreto de 13 de abril de 1962 que estableció la composición de los comités profesionales encargados de elaborar los Planes Contables Profesionales, que es como se denominarian las adaptaciones sectoriales. En aplicación de dicha ley, se realizaron más de un centenar de adaptaciones del Plan Contable General 1957, algunas de las cuales fueron adaptaciones completas y otras simples recomendaciones sobre distintos aspectos contables. Una de las adaptaciones realizadas fue el Plan Contable Profesional de la Industria Hotelera (Plan Comptable Professionnel de l'ndustrie Hóteliére), aprobado por decreto de 9 de octubre de 1970.

La autora a continuación se lamentaba de la ausencia de un Plan Contable profesional similar en España. En esta línea y en el mismo trabajo doctoral ofrecía todo un Plan Contable sectorial aplicable al caso español. Venía a sumarse así a otro histórico intento de adaptación sectorial ambiciosamente propuesto por José María Arespacochaga en los años 1980. La misma autora tampoco disimulaba su sorpresa del escaso éxito que consiguió una propuesta auspiciada por la Asociación Nacional de Directores de Hotel. Por circunstancias que serían interesantes analizar no llegó a cristalizar, a pesar del respaldo que recibió del Instituto Español de Turismo y de la Federaciones de Empresarios Hoteleros (Vacas 1999, pp. 420). En su presentación el autor afirmaba lo siguiente:

"El Plan que presentamos cubre la triple dimensión que creemos necesaria en la industria botel era atendiendo a las necesidades de ésta en cuanto a contabilidad financiera o general, en cuanto a contabilidad analítica o de costes por centros de explotación y en cuanto a contabilidad presupuestaria que permita controlar no ya un presupuesto de explotación sino su programa completo de actuaciones, financiación e inversiones".

No parece que pueda negarse a esta propuesta su entronque con la tradición de los Uniform Systems, salvo en un detalle: los Uniform System anglosajones son una exclusiva iniciativa particular del sector y no una obligación impuesta desde la autoridad pública. Este punto, junto con el énfasis en la contabilidad de gestión sobre la financiera, explicaría la vitalidad demostrada por el USALI en sus 90 años de vigencia.

Vacas (1999, pp. 419) concluía su extenso estudio abogando por "una adaptación del Plan General de Contabilidad a la industria de la hostelería", consciente, sin embargo, de la multiplicidad de modelos normativos por elegir:

Unas veces la normalización ha estado promovida por la iniciativa privada y otras de la pública; en ocasiones se ha aplicado con carácter general a todas las empresas y en otras no; hay quien opina que las normas deben ser simples recomendaciones de aceptación voluntaria y quien dice que deben imponerse mediante una disposición 
legal; sin olvidar la posición intermedia, de los partidarios de que no sea obligatoria, pero que se incentive a las empresas que las aplican mediante ciertas ventajas.

El reto que al directivo español presenta la tradición de los sistemas uniformes para hoteles está suficientemente perfilado con sólo poner la vista atrás, reconocer el camino andado, especialmente en el mundo anglosajón y ver, además, la importancia económica del sector hotelero.

En este sentido, el sector turístico es una de las actividades económicas más importantes de la economía española. Genera una gran cantidad de riqueza y empleo, y tiene una importante cuota de participación en la economía nacional. El mercado español, aunque es muy dependiente del comportamiento de los factores determinantes del consumo exterior, fundamentalmente el que se refiere a Europa ha conseguido consolidarse como uno de los mercados relevantes a nivel mundial. El sector hotelero forma un sector fundamental en una economía como la española. De hecho España consigue unos muy buenos resultados en el sector del turismo, alcanza durante el año 2015 unos 68 millones de entradas de turistas (según datos del Instituto Nacional de Estadística). Por tipo de alojamiento, el de mercado fue utilizado por 53.1 millones de turistas (un 5.4\% más) -de los que 43.1 millones (un 4.9\% más) pernoctaron en hoteles, un $63.3 \%$ del total-. Cataluña fue la principal comunidad de destino en 2015, seguida de Baleares y Canarias mientras que Madrid fue la que más creció.

Por otra parte la hotelería tiene unas características propias del sector; el producto o servicio ofrecido es fugaz, la calidad de la prestación del servicio al cliente es un atributo clave, existen una gran variedad de productos y servicios que son prestados u ofrecidos de forma conjunta, la empresa debe satisfacer las necesidades de los clientes 24 horas al día durante todos los días del año, el producto se consume en el mismo lugar de producción, existen múltiples fuentes de ingresos como consecuencia de los diversos centros de beneficios existentes en la empresa hotelera (habitaciones, restaurantes, tiendas, etc.). En aquellos casos en que coincide propiedad con gestión la inversión en activos no corrientes suele ser elevada. En los casos en que la empresa que gestiona el hotel no es propietaria del mismo esa inversión a largo plazo es menos significativa. Los indicadores utilizados son específicos de los hoteles.

Cuando hay un sector del país que reúna dos características principalmente; ser relevante para la economía del país y tener características propias del sector, es cuando surge la necesidad de elaborar un PGC sectorial. Un PGC sectorial que serviría para tener un sistema uniforme de contabilidad demandado por sus usuarios. 
De la información manejada hasta aquí parece clara una conclusión: los sistemas uniformes de contabilidad es bueno que se fomenten porque siguen en pie las necesidades y lagunas que los actores de principios del siglo XX ya detectaron. Gerhard G. Mueller (1967) recuerda significativamente una figura capital en la historia de la contabilidad europea:

Seen internationally, the desire to achieve greater uniformity in accounting has been with us for a long time. In the early 1920s, the German Professor Eugen Schmalenbach was frustrated again and again by the failure of bis own efforts and those of his students to compare meaningfully the financial data made available by different companies. This led to concentrated research on the problem and resulted in the publication of a book by Schmalenbach entitled The Model Chart of Accounts. With this book Schmalenbach laid the foundation for all subsequent developments in uniform accounting in Germany.

En general convendrá tener presente la observación final contenida en el libro de Littleton (1966) en torno a la evolución de los sistemas contables y de la contabilidad en general: "It came from definite causes; it moves toward a definite destin".

\section{Referencias}

Berk, G. (1997). Discursive Cartels: Uniform Cost Accounting Among American Manufacturers Before the New Deal. Business And Economic History, 26(1), 229-251. University of Oregon: Business History Conference. ISSN 0894-682.

Bouquin, H. (2003). La comptabilité de gestion. Col: «Que sais-je?» n 3175. París: PUF-Presses Universitaires de France.

Calmès, A. (1922). La comptabilité industrielle. Paris: Ed. Payot.

Campa, F., \& Amat, O. (2005). La importancia de la contabilidad de gestión en el sector hotelero. Revista de la Asociación Española de Contabilidady Administración de Empresas, 72, 109.

Campa, F., \& Rebull, M. (2007). Un análisis de las prácticas en contabilidad de gestión dentro del sector hotelero: Diferencias entre el segmento vacacional y el de ciudad. Revista Iberoamericana de Contabilidad de Gestión, 9, 159-184.

Dragos, Z. (2009). Les multiples enjeux d'une technique de gestion: pratiques et discours dans la répartition des frais généraux. Université d'Orléans: Tesis doctoral.

Faria, A. (2012). Sistemas de Contabilidade de Gestão no Setor Hoteleiro do Algarve: Planeamento ou Improvisação?. Tese de Mestrado. Universidade do Algarve. 
Johnson, H.T., \& Kaplan, R.H. (1987). Relevance Lost. Boston: Ed. H.B.S.

Julhiet, E. (1922). Cours de finance et comptabilité dans l'industrie. Paris: Ed. Eyrolles.

Littleton, A.C. (1966). Accounting Evolution to 1900. New-York: Russel and Russel.

Mueller, G.G. (1967). International accounting (Part 1). New York: Ed. Macmillan.

NACA - National Association of Cost Accountants (1921). Year Book. New York

Nielsen, O. (1966). A predecessor of Direct Costing. Journal of Accounting Research, 4(1), 119-120. https://doi.org/10.2307/2490145

Nikitin, M. (1992). La naissance de la comptabilité industrielle en France. Tesis en ciencias de gestión, Université Paris-Dauphine.

Palewski, J.P. (1944). L'organisation scientifique du travail. Col: « Que sais-je ?» n 31. Paris: PUF.

Schmidgall, R.S. (2014). Evolution of the Uniform System of Accounts for the Lodging Industry. The Journal of Hospitality Financial Management. 22(1), 41-57. https://doi.org/10.1080/10913211.2014.912105

Trossen, J. (1934). Le calcul des prix de revient industriels. Universidad de Lovaina.

Vacas Guerrera, C. (1999). Normalización contable del sector de la hostelería. Una propuesta de adaptación del plan general de contabilidad al sector. Tesis doctoral. Universidad Complutense de Madrid.

Zimnovitch, H. (1997). Les calculs du prix de revientdans la seconde industrialisation en France. Business administration. Universidad de Poitiers.

Intangible Capital, 2017 (www.intangiblecapital.org)

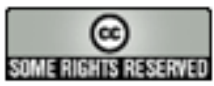

Article's contents are provided on an Attribution-Non Commercial 3.0 Creative commons license. Readers are allowed to copy, distribute and communicate article's contents, provided the author's and Intangible Capital's names are included. It must not be used for commercial purposes. To see the complete license contents, please visit http://creativecommons.org/licenses/by-nc/3.0/. 